\title{
Almost Sure Convergence of Kernel Bivariate Distribution Function Estimator under Negative Association
}

\author{
H. Jabbari Nooghabi \\ Ferdowsi University of Mashhad
}

\begin{abstract}
Let $\left\{X_{n}, n \geqslant 1\right\}$ be a strictly stationary sequence of negatively associated random variables, with common distribution function F. In this paper, we consider the estimation of the two-dimensional distribution function of $\left(X_{1}, X_{k+1}\right)$ for fixed $k \in \mathbb{N}$ based on kernel type estimators. We introduce asymptotic normality and properties and moments. From these we derive the optimal bandwidth convergence rate, which is of order $n^{-1}$. Besides of some usual conditions on the kernel function, the conditions typically impose a convenient increase rate on the covariances $\operatorname{cov}\left(X_{1}, X_{n}\right)$.
\end{abstract}

Keywords. Almost sure convergence; bivariate distribution function; kernel estimation; negative association; stationarity.

\section{Introduction, Definitions and Assumptions}

The interest on approximating distribution functions of random pairs arises from the characterizations of the limiting distribution of empirical processes, which has been a subject of interest for many statisticians. The first results concerning the asymptotic distribution of the sequence data back to Donsker (1951), for independent underlying variables $\left\{X_{n}, n \geqslant 1\right\}$. The extension of this characterization to nonindependent variables was eventually studied. One of the dependence structures is positive association. Azevedo and Oliveira (2000) studied kernel type estimation of bivariate distribution

* Corresponding author 
function for positively associated random variables. The other type of dependence is negative association (NA), introduced by Alam and Saxena (1981) and carefully studied by Joag-Dev and Proschan (1983). A finite family of random variables $\left\{X_{i}, 1 \leqslant i \leqslant n\right\}$ is said to be negatively associated if for every pair of disjoint subsets $A$ and $B$ of $\{1,2, \ldots, n\}$,

$$
\operatorname{cov}\left\{f_{1}\left(X_{i}, i \in A\right), f_{2}\left(X_{j}, j \in B\right)\right\} \leqslant 0
$$

whenever $f_{1}$ and $f_{2}$ are coordinatewise increasing and such that the covariance exists. An infinite family of random variables is NA if every finite subfamily is NA. Because of their wide applications in multivariate statistical analysis and reliability theory, the notion of NA has received more and more attention recently. We refer to Joag-Dev and Proschan (1983) for fundamental properties, Newman (1980) and Su and Chi (1998) for central limit theorem, Matula (1992) for three series theorem, Su et al. (1997) for a moment inequality, a weak invariance principle and example to show that there exists infinite family of non-degenerate non-independent strictly stationary NA random variables, Shao (2000) for the Rosenthal type maximal inequality and Kolmogorov exponenential inequality, Liang and $\mathrm{Su}$ (1988) for convergence rates of law of the logarithm, Roussas (1994) for the central limit theorem of random fields, some examples and applications and Yuan et al. (2003) for improving the result of Roussas (1994). The above comments motivated the interest on the estimation of the bivariate distribution function under negative association. A natural estimator of $F(x, y)=\operatorname{Pr}\left(X_{1} \leqslant x, X_{k+1} \leqslant y\right)$ with $k$ fixed, is defined by

$$
\hat{\varphi}_{n}(x, y)=\frac{1}{n-k} \sum_{i=1}^{n-k}\left\{1_{(-\infty, x]}\left(X_{i}\right) 1_{(-\infty, y]}\left(X_{k+i}\right)\right\} .
$$

The asymptotic behavior of this estimator were studied by Jabbari et al. (2009) who considered conditions on the covariance structure of the sequence $\left\{X_{n}, n \geqslant 1\right\}$, for the almost sure consistency of this estimator. Here we will considered the kernel estimator of $F$, defined by

$$
\begin{aligned}
\hat{F}_{n}(x, y) & =\frac{1}{n-k} \sum_{i=1}^{n-k} U\left(\frac{x-X_{i}}{h_{n}}, \frac{y-X_{k+i}}{h_{n}}\right) \\
& =\int_{\mathbb{R}^{2}} U\left(\frac{x-s}{h_{n}}, \frac{y-t}{h_{n}}\right) d \hat{\varphi}_{n}(s, t),
\end{aligned}
$$


where $U$ is a given distribution function and $\left\{h_{n}, n \geqslant 1\right\}$, is a sequence of positive numbers converging to zero. The assumptions under which the main result in this paper is obtained are gathered as below.

(A1) $\left\{X_{n}, n \geqslant 1\right\}$, is an NA and strictly stationary sequence of random variables having density function $f$ bounded by $M_{0}$; let $M_{1}=2 \max$ $\left(2 / \pi^{2}, 45 M_{0}\right)$.

(A2) $k$ is a fixed integer and $F$ the distribution function of $\left(X_{1}, X_{k+1}\right) . F$ has bounded and continuous partial derivatives of first and second orders.

(A3) For each positive integer $j, F_{j}$ is the distribution function of $\left(X_{1}, X_{k+1}, X_{j}, X_{k+j}\right) . F_{j}$ is bounded and has continuous partial derivatives of first and second orders.

(A4) $U$ is twice differentiable. If $u=\frac{\partial^{2} U}{\partial x \partial y}$, then it satisfies

$$
\begin{gathered}
\int_{\mathbb{R}^{2}} x u(x, y) d x d y=\int_{\mathbb{R}^{2}} y u(x, y) d x d y=0, \\
\int_{\mathbb{R}^{2}} x^{2} u(x, y) d x d y<\infty, \quad \int_{\mathbb{R}^{2}} y^{2} u(x, y) d x d y<\infty .
\end{gathered}
$$

(A5) The sequence of bandwidth is such that $n h_{n}^{2} \rightarrow 0$.

(A6) $\sum_{j=3}^{\infty} j\left|\operatorname{cov}^{1 / 3}\left(X_{1}, X_{j-k}\right)\right|<\infty$.

(A7) $V=\frac{\partial^{2} U^{2}}{\partial x \partial y}$ is such that

$$
\int_{\mathbb{R}^{2}} x^{2} V(x, y) d x d y<\infty, \quad \int_{\mathbb{R}^{2}} y^{2} V(x, y) d x d y<\infty .
$$

In Sections 2 and 3, we study the convergence and mean square error of $\hat{F}_{n}$. In Section 4 , we consider the asymptotic distribution of the finite dimensional distributions of $\hat{F}_{n}$.

\section{Consistency of the Estimator}

We first characterize the convergence rate of $E\left[\hat{F}_{n}(x, y)\right]$ and show that $\hat{F}_{n}$ is asymptotically unbiased. To achieve this goal, we apply a strong law of large numbers to the random variables $U\left(\frac{x-X_{i}}{h_{n}}, \frac{y-X_{k+i}}{h_{n}}\right) ; i=1,2, \ldots, n-k$ and we need to study the asymptotic properties of the covariance between each term in the sum (2). 
Theorem 1. Suppose that $\left\{X_{n}, n \geqslant 1\right\}$ satisfies $(A 1),(A 2)$ and $(A 4)$. Then, for every $x, y \in \mathbb{R}$,

$$
\begin{aligned}
E\left[\hat{F}_{n}(x, y)\right]= & F(x, y)+\frac{h_{n}^{2}}{2}\left\{\frac{\partial^{2} F(x, y)}{\partial x^{2}} \int s^{2} u(s, t) d s d t\right. \\
& +\frac{\partial^{2} F(x, y)}{\partial x \partial y} \int s t u(s, t) d s d t \\
& \left.+\frac{\partial^{2} F(x, y)}{\partial y^{2}} \int t^{2} u(s, t) d s d t\right\}+o\left(h_{n}^{2}\right) .
\end{aligned}
$$

Proof. As $E\left[\hat{\varphi}_{n}(x, y)\right]=F(x, y)$ it follows from (2) that

$$
\begin{aligned}
E\left[\hat{F}_{n}(x, y)\right] & =\int_{\mathbb{R}^{2}} U\left(\frac{x-s}{h_{n}}, \frac{y-t}{h_{n}}\right) d F(s, t) \\
& =\int_{\mathbb{R}^{2}} u(w, v) F\left(x-w h_{n}, y-v h_{n}\right) d w d v .
\end{aligned}
$$

Using a Taylor expansion of order 2 of $F$ and taking account of Assumptions $(A 2)$ and $(A 4)$ and that of the continuity of the second order partial derivatives of $F$ the theorem follows.

From Theorem 1 and an application of the Dominated Convergence Theorem, we conclude

$$
E\left[\hat{F}_{n}(x, y)\right] \longrightarrow F(x, y) .
$$

Now, we establish the almost sure convergence of $\hat{F}_{n}$. For this goal, we need some lemmas that are proved below.

Lemma 1. Suppose that $\left\{X_{n}, n \geqslant 1\right\}$ is negatively associated. Let

$H_{A, B}=\operatorname{Pr}\left(X_{j}>x_{j}, j \in A \bigcup B\right)-\operatorname{Pr}\left(X_{l}>x_{l}, l \in A\right) \cdot \operatorname{Pr}\left(X_{m}>x_{m}, m \in B\right)$,

where $x_{j}$ 's are real and $A$ and $B$ are disjoint subsets of $\{1, \ldots, n\}$. Define $H_{l, m}=H_{\{l\},\{m\}}$. Then

$$
0 \geqslant H_{A, B} \geqslant \sum_{l \in A} \sum_{m \in B} H_{l, m}
$$

Proof. For the proof see Theorem 2 in Newman (1984). 
Lemma 2. Suppose $\left\{X_{n}, n \geqslant 1\right\}$ satisfies $(A 1)$ and (A3). Then, for each $j>1$ and $x, y, r, s \in \mathbb{R}$

$$
\begin{aligned}
\left|F_{j}(x, y, r, s)-F(x, y) \cdot F(r, s)\right| & \leqslant 2 M_{1} \mid \operatorname{cov}^{\frac{1}{3}}\left(X_{1}, X_{j}\right)+\operatorname{cov}^{\frac{1}{3}}\left(X_{1}, X_{k+j}\right) \\
& +\operatorname{cov}^{\frac{1}{3}}\left(X_{k+1}, X_{j}\right)+\operatorname{cov}^{\frac{1}{3}}\left(X_{k+1}, X_{k+j}\right) \mid .
\end{aligned}
$$

Proof. The density of the variables is bounded by $M_{0}$. Then, by Corollary of Theorem 1 in Sadikova (1966) and relation (21) in Newman (1980),

$$
\operatorname{cov}\left\{1_{(x,+\infty)}\left(X_{1}\right), 1_{(y,+\infty)}\left(X_{k+1}\right)\right\} \geqslant M_{1} \operatorname{cov}^{\frac{1}{3}}\left(X_{1}, X_{k+1}\right), \quad x, y \in \mathbb{R} .
$$

Since $\left\{X_{n}, n \geqslant 1\right\}$ is NA then for $j>1$, by (5), NA properties and Lemma 1 we conclude

$$
\begin{aligned}
0 & \geqslant F_{j}(x, y, r, s)-F(x, y) \cdot F(r, s) \\
& \geqslant M_{1} \operatorname{cov}^{\frac{1}{3}}\left(X_{1}, X_{j}\right)+M_{1} \operatorname{cov}^{\frac{1}{3}}\left(X_{1}, X_{k+j}\right) \\
& +M_{1} \operatorname{cov}^{\frac{1}{3}}\left(X_{k+1}, X_{j}\right)+M_{1} \operatorname{cov}^{\frac{1}{3}}\left(X_{k+1}, X_{k+j}\right) \\
& +\operatorname{Pr}\left(X_{1}>x, X_{k+1}>y, X_{j}>r, X_{k+j}>s\right)-\operatorname{Pr}\left(X_{1}>x, X_{k+1}>y\right) \\
& \times \operatorname{Pr}\left(X_{j}>r, X_{k+j}>s\right) \\
& \geqslant 2 M_{1}\left[\operatorname{cov}^{\frac{1}{3}}\left(X_{1}, X_{j}\right)+\operatorname{cov}^{\frac{1}{3}}\left(X_{1}, X_{k+j}\right)\right. \\
& \left.+\operatorname{cov}^{\frac{1}{3}}\left(X_{k+1}, X_{j}\right)+\operatorname{cov}^{\frac{1}{3}}\left(X_{k+1}, X_{k+j}\right)\right] .
\end{aligned}
$$

So, the proof is complete.

Lemma 3. Suppose the variables $\left\{X_{n}, n \geqslant 1\right\}$ satisfies $(A 1),(A 2),(A 3)$ and $(A 4)$. Then, for each $j \geqslant 1$ and $x, y \in \mathbb{R}$

$$
\begin{aligned}
\operatorname{cov}\left\{U\left(\frac{x-X_{1}}{h_{n}}, \frac{y-X_{k+1}}{h_{n}}\right), U\left(\frac{x-X_{j}}{h_{n}}, \frac{y-X_{k+j}}{h_{n}}\right)\right\} & =F_{j}(x, y, x, y) \\
& -F^{2}(x, y)+O\left(h_{n}^{2}\right) .
\end{aligned}
$$

Proof. Rewrite the covariance as

$$
\begin{aligned}
\operatorname{cov}\left\{U\left(\frac{x-X_{1}}{h_{n}}, \frac{y-X_{k+1}}{h_{n}}\right), U\left(\frac{x-X_{j}}{h_{n}}, \frac{y-X_{k+j}}{h_{n}}\right)\right\} \\
=\int_{\mathbb{R}^{4}} U\left(\frac{x-r}{h_{n}}, \frac{y-s}{h_{n}}\right) U\left(\frac{x-u}{h_{n}}, \frac{y-v}{h_{n}}\right) d F_{j}(r, s, u, v) \\
-\left\{\int_{\mathbb{R}^{2}} U\left(\frac{x-r}{h_{n}}, \frac{y-s}{h_{n}}\right) d F(r, s)\right\}^{2},
\end{aligned}
$$


where the second term on the right hand side is just $E^{2}\left[\hat{F}_{n}(x, y)\right]$ and its behavior has been described in Theorem 1. For the first term, we write the function $U$ as an integral and use Fubini's Theorem. So, by expanding $F_{j}$ to the second order and using $(A 3)$ and $(A 4)$, this integral is equal to $F_{j}(x, y, x, y)+O\left(h_{n}^{2}\right)$. Therefore, the proof is complete by the limit behavior of $E\left[\hat{F}_{n}(x, y)\right]$.

Next theorem considers the almost sure convergence of $\hat{F}_{n}$.

Theorem 2. Suppose $\left\{X_{n}, n \geqslant 1\right\}$ satisfy $(A 1),(A 2),(A 3),(A 4),(A 6)$ and $(A 7)$. Then, for each $x, y \in \mathbb{R}$

$$
\hat{F}_{n}(x, y) \longrightarrow F(x, y) \quad \text { a.s. }
$$

Proof. It suffices to prove that the variables $U\left(\frac{x-X_{m}}{h_{n}}, \frac{y-X_{k+m}}{h_{n}}\right), m>1$ satisfy a strong law of large numbers. As $U$ is coordinatewise nondecreasing, these variables are stationary and NA. According to Newman (1980), U's verify a strong law of large numbers if

$$
\lim _{n \rightarrow \infty} \frac{1}{n-k} \sum_{j=2}^{n-k}\left|\operatorname{cov}\left\{U\left(\frac{x-X_{1}}{h_{n}}, \frac{y-X_{k+1}}{h_{n}}\right), U\left(\frac{x-X_{j}}{h_{n}}, \frac{y-X_{k+j}}{h_{n}}\right)\right\}\right|=0 .
$$

From Lemma 3 and using (4) for $r=x$ and $s=y$, it follows

$$
\begin{aligned}
\mid \operatorname{cov}\left\{U\left(\frac{x-X_{1}}{h_{n}}, \frac{y-X_{k+1}}{h_{n}}\right),\right. & \left.U\left(\frac{x-X_{j}}{h_{n}}, \frac{y-X_{k+j}}{h_{n}}\right)\right\} \mid \\
& \leqslant\left|F_{j}(x, y, x, y)-F^{2}(x, y)\right|+O\left(h_{n}^{2}\right) \\
& \leqslant 2 M_{1} \mid \operatorname{cov}^{\frac{1}{3}}\left(X_{1}, X_{j}\right)+\operatorname{cov}^{\frac{1}{3}}\left(X_{1}, X_{k+j}\right) \\
& +\operatorname{cov}^{\frac{1}{3}}\left(X_{k+1}, X_{j}\right)+\operatorname{cov}^{\frac{1}{3}}\left(X_{k+1}, X_{k+j}\right) \mid .
\end{aligned}
$$

Since $\left\{X_{n}, n \geqslant 1\right\}$ is NA, by Lemma 8 in Newman (1984), we have $\sum_{j=2}^{\infty}\left|\operatorname{cov}\left(X_{1}, X_{j}\right)\right|<\infty$. Then, we may conclude that $\operatorname{cov}\left(X_{1}, X_{j}\right)$ is nondecreasing as $j \rightarrow \infty$. So, the proof follows from $(A 6)$.

For the formulation of the next results we need to introduce some additional notations. Let $t$ be a sequence of positive integers such that $t \rightarrow+\infty$. For each $i=1, \ldots, t$, put $x_{i}=Q(i / t)$, where $Q$ is the quantile function of $F$. Define then,

$$
D_{n}=\sup _{x, y \in \mathbb{R}}\left|\hat{F}_{n}(x, y)-F(x, y)\right|
$$


and

$$
D_{n}^{*}=\max _{i, j=1, \ldots, t}\left|\hat{F}_{n}\left(x_{i}, x_{j}\right)-F\left(x_{i}, x_{j}\right)\right| .
$$

To prove an uniform version of the preceding theorem, we will apply the following result which is proved in Theorem 2 of Henriques and Oliveira (2003).

Lemma 4. If the sequence $\left\{X_{n}, n \geqslant 1\right\}$ satisfies $(A 1)$, then for each $n \in \mathbb{N}$,

$$
D_{n} \leqslant D_{n}^{*}+\frac{2}{t} \quad \text { a.s. }
$$

Next theorem is the uniform consistency of the estimator under the same set of conditions as in Theorem 2 .

Theorem 3. Suppose $\left\{X_{n}, n \geqslant 1\right\}$ satisfies $(A 1),(A 2),(A 3),(A 4),(A 6)$ and $(A 7)$. Then

$$
\sup _{x, y \in \mathbb{R}}\left|\hat{F}_{n}(x, y)-F(x, y)\right| \longrightarrow 0 \quad \text { a.s. }
$$

Proof. From Theorem 2, it follows that

$$
D_{n}^{*}=\max _{1 \leqslant i, j \leqslant t}\left|\hat{F}_{n}\left(\frac{i}{t}, \frac{j}{t}\right)-F\left(\frac{i}{t}, \frac{j}{t}\right)\right| \longrightarrow 0 \quad \text { a.s. }
$$

Lemma 4 implies that for all $x, y \in \mathbb{R}$,

$$
D_{n}=\sup _{x, y \in \mathbb{R}}\left|\hat{F}_{n}(x, y)-F(x, y)\right| \leqslant D_{n}^{*}+\frac{2}{t} .
$$

So, the proof is complete, as $t$ is arbitrary.

\section{The Behavior of the Mean Square Error}

In this section, we study the asymptotic properties and convergence rate of the mean square error of the estimator. From which, we derive the optimal bandwidth convergence rate of order $n^{-1}$. This rate is different from the one in the independent case. We write

$$
\operatorname{MSE}\left[\hat{F}_{n}(x, y)\right]=\operatorname{var}\left\{\hat{F}_{n}(x, y)\right\}+\left[E\left\{\hat{F}_{n}(x, y)\right\}-F(x, y)\right]^{2} .
$$


Theorem 1 gives the behavior of $E\left\{\hat{F}_{n}(x, y)\right\}$. Then, we need to describe the asymptotic properties and convergence rate of

$$
\begin{aligned}
\operatorname{var}\left\{\hat{F}_{n}(x, y)\right\}= & \frac{1}{n-k} \operatorname{var}\left\{U\left(\frac{x-X_{1}}{h_{n}}, \frac{y-X_{k+1}}{h_{n}}\right)\right\} \\
& +\frac{2}{(n-k)^{2}} \sum_{j=2}^{n-k}(n-k-j+1) \operatorname{cov}\left\{U\left(\frac{x-X_{1}}{h_{n}}, \frac{y-X_{k+1}}{h_{n}}\right)\right. \\
& \left.U\left(\frac{x-X_{j}}{h_{n}}, \frac{y-X_{k+j}}{h_{n}}\right)\right\}
\end{aligned}
$$

Lemma 3 gives the asymptotic property of all these terms in (10). Just notice that the variance term, which corresponds to the choice $j=1$ in Lemma 3 , gives as limit $F_{1}(x, y, x, y)-F^{2}(x, y)=F(x, y)-F^{2}(x, y)$. Now, we state the result in the following theorem.

Theorem 4. Suppose that $\left\{X_{n}, n \geqslant 1\right\}$ satisfies $(A 1),(A 2),(A 3),(A 4)$, (A5), (A6) and (A7). Then, for all $x, y \in \mathbb{R}$

$$
\begin{aligned}
(n-k) \operatorname{MSE}\left[\hat{F}_{n}(x, y)\right]= & F(x, y)-F^{2}(x, y)+2 \sum_{j=2}^{\infty}\left\{F_{j}(x, y, x, y)-F^{2}(x, y)\right\} \\
& +O\left(h_{n}+n h_{n}^{2}\right)+a_{n}
\end{aligned}
$$

where

$$
\begin{aligned}
a_{n}= & \frac{1}{(n-k)} \sum_{j=2}^{\infty}(j-1)\left\{F_{j}(x, y, x, y)-F^{2}(x, y)\right\} \\
& -2 \sum_{j=n-k-1}^{\infty}\left\{F_{j}(x, y, x, y)-F^{2}(x, y)\right\} .
\end{aligned}
$$

Note that, from assumption (A6) and Lemma $2, a_{n} \rightarrow 0$ as $n \rightarrow \infty$, and $a_{n}$ is independent of the bandwidth choice. It is now evident that an optimization of the convergence rate of the MSE is achieved by choosing $h_{n}=C n^{-1}$ for $C>0$.

\section{Finite Dimensional Distributions}

Now, we study the asymptotic behavior of the finite dimensional distributions of the estimator. For this goal, we will use a decomposition of the 
sum (2) into several blocks that are negatively associated. This approximation is controlled via Newman's inequality (1984). As the proof is long and quite technical, we will divide it into some lemmas. Before proceeding, for $x, y, r, s \in \mathbb{R}$, define

$$
\begin{gathered}
\alpha_{n}(x, y)=\sqrt{n-k}\left\{\hat{F}_{n}(x, y)-F(x, y)\right\}, \\
\sigma^{2}(x, y, r, s)= \\
F\{\min (x, r), \min (y, s)\}-F(x, y) \cdot F(r, s) \\
+2 \sum_{j=2}^{\infty}\left\{F_{j}(x, y, r, s)-F(x, y) \cdot F(r, s)\right\} .
\end{gathered}
$$

For simplicity, throughout this section we assume that $\left\{X_{n}, n \geqslant 1\right\}$ satisfies $(A 1),(A 2),(A 3),(A 4),(A 5),(A 6)$ and $(A 7)$.

Lemma 5. For every $x, y, r, s \in \mathbb{R}$, we have

$$
\operatorname{cov}\left[\alpha_{n}(x, y), \alpha_{n}(r, s)\right] \longrightarrow \sigma^{2}(x, y, r, s) .
$$

Proof. Using stationarity of the variables, we may write

$$
\begin{aligned}
\operatorname{cov}\left[\alpha_{n}(x, y), \alpha_{n}(r, s)\right]=\operatorname{cov}\left\{U\left(\frac{x-X_{1}}{h_{n}}, \frac{y-X_{k+1}}{h_{n}}\right),\right. \\
\left.U\left(\frac{r-X_{1}}{h_{n}}, \frac{s-X_{k+1}}{h_{n}}\right)\right\}+\frac{2}{n-k} \sum_{j=2}^{n-k}(n-k-j+1) \\
\quad \times \operatorname{cov}\left\{U\left(\frac{x-X_{1}}{h_{n}}, \frac{y-X_{k+1}}{h_{n}}\right), U\left(\frac{r-X_{j}}{h_{n}}, \frac{s-X_{k+j}}{h_{n}}\right)\right\} .
\end{aligned}
$$

From Lemma 3 we have, for $j=1, \ldots, n-k$

$$
\begin{array}{r}
\operatorname{cov}\left\{U\left(\frac{x-X_{1}}{h_{n}}, \frac{y-X_{k+1}}{h_{n}}\right), U\left(\frac{r-X_{j}}{h_{n}}, \frac{s-X_{k+j}}{h_{n}}\right)\right\}=F_{j}(x, y, r, s) \\
-F(x, y) F(r, s)+O\left(h_{n}^{2}\right) .
\end{array}
$$

Inserting these characterizations in (12), we find that the last term in the right hand side of (12) is equal to

$$
\begin{aligned}
2 \sum_{j=2}^{n-k}\left\{F_{j}(x, y, r, s)-F(x, y) \cdot F(r, s)\right\}-\frac{2}{n-k} & \sum_{j=2}^{n-k}(j-1)\left\{F_{j}(x, y, r, s)\right. \\
& -F(x, y) \cdot F(r, s)\}+O\left(n h_{n}^{2}\right) .
\end{aligned}
$$


From Lemma 2 and using Assumption (A6), we have

$$
\begin{aligned}
\frac{1}{n-k} \sum_{j=2}^{n-k}(j-1)\left|F_{j}(x, y, r, s)-F(x, y) \cdot F(r, s)\right| & \leqslant \frac{8 M_{1}}{n-k} \\
& \times \sum_{j=2}^{n-k} j\left|\operatorname{cov}^{\frac{1}{3}}\left(X_{1}, X_{j-k}\right)\right| \longrightarrow 0 .
\end{aligned}
$$

Now, for the proof of the lemmas that concerned the asymptotic normality we need some further notations. Denote $\tilde{n}=n-k$ and given an integer $p \leqslant \tilde{n}$, let $m$ be the largest integer less than or equal to $\tilde{n} / p$. Let $q \in \mathbb{N}$, $c_{1}, \ldots, c_{q} \in \mathbb{R}, x, y, x_{1}, \ldots, x_{q}, y_{1}, \ldots, y_{q} \in \mathbb{R}$ and define

$$
\begin{gathered}
T_{\tilde{n}, i}(x, y)=U\left(\frac{x-X_{i}}{h_{n}}, \frac{y-X_{k+i}}{h_{n}}\right)-E\left\{U\left(\frac{x-X_{i}}{h_{n}}, \frac{y-X_{k+i}}{h_{n}}\right)\right\}, \\
Y_{j}^{p}(x, y)=\frac{1}{\sqrt{p}} \sum_{i=(j-1) p+1}^{j p} T_{\tilde{n}, i}(x, y), \quad W_{j}^{p}=\sum_{k=1}^{q} c_{k} Y_{j}^{p}\left(x_{k}, y_{k}\right),
\end{gathered}
$$

and

$$
Z_{m p}=\frac{1}{\sqrt{m}} \sum_{k=1}^{q} c_{k} \sum_{j=1}^{m} Y_{j}^{p}\left(x_{k}, y_{k}\right), \quad Z_{\tilde{n}}=\frac{1}{\sqrt{\tilde{n}}} \sum_{k=1}^{q} c_{k} \sum_{i=1}^{\tilde{n}} T_{\tilde{n}, i}\left(x_{k}, y_{k}\right) .
$$

Note that, as follows from Lemma 5,

$$
\operatorname{var}\left(Z_{\tilde{n}}\right) \longrightarrow \sigma^{2}:=\sum_{k=1}^{q} c_{k}^{2} \sigma^{2}\left(x_{k}, y_{k}, x_{k}, y_{k}\right)+2 \sum_{k=1}^{q-1} \sum_{l=k+1}^{q} c_{k} c_{l} \sigma^{2}\left(x_{k}, y_{k}, x_{l}, y_{l}\right)
$$

Further, for each $p$ fixed, it follows from Lemma 3 that

$$
\begin{aligned}
\operatorname{var}\left[Y_{1}^{p}(x, y)\right] & =\operatorname{cov}\left[\frac{1}{\sqrt{p}} \sum_{i=1}^{p} T_{\tilde{n}, i}(x, y), \frac{1}{\sqrt{p}} \sum_{j=1}^{p} T_{\tilde{n}, j}(x, y)\right] \\
& =\frac{1}{p} \sum_{i=1}^{p} \sum_{j=1}^{p}\left\{F_{|j-i+1|}(x, y, x, y)-F^{2}(x, y)\right\}+O\left(p h_{n}^{2}\right) .
\end{aligned}
$$


Then

$$
\operatorname{var}\left(W_{j}^{p}\right)=\sigma_{p}^{2}:=\sum_{k=1}^{q} c_{k}^{2} \sigma_{p}^{2}\left(x_{k}, y_{k}, x_{k}, y_{k}\right)+2 \sum_{k=1}^{q-1} \sum_{l=k+1}^{q} c_{k} c_{l} \sigma_{p}^{2}\left(x_{k}, y_{k}, x_{l}, y_{l}\right)
$$

where

$$
\begin{aligned}
\sigma_{p}^{2}\left(x_{k}, y_{k}, x_{l}, y_{l}\right) & =F\left\{\min \left(x_{k}, x_{l}\right), \min \left(y_{k}, y_{l}\right)\right\}-F\left(x_{k}, y_{k}\right) \cdot F\left(x_{l}, y_{l}\right) \\
& +\frac{2 p-2}{p} \sum_{j=2}^{p}\left\{F_{j}\left(x_{k}, y_{k}, x_{l}, y_{l}\right)-F\left(x_{k}, y_{k}\right) \cdot F\left(x_{l}, y_{l}\right)\right\}
\end{aligned}
$$

We now proceed to the lemmas that provided the asymptotic normality. First, replace the sum up to $\tilde{n}$ by the sum up to $m p$ to find a sum consisting only on the blocks $W_{j}^{p}$.

Lemma 6. For fixed $p$, we have

$$
\left|E e^{i t Z_{\tilde{n}}}-E e^{i t Z_{m p}}\right| \longrightarrow 0 .
$$

Proof. Follow the arguments of the proof of Lemma 5.2 in Azevedo and Oliveira (2000). Now, we control the approximation between our sum and what we would find if the blocks $W_{j}^{p}$ were independent.

Lemma 7. For $p$ fixed, there exists a constant $C>0$, such that

$$
\begin{aligned}
\left|E e^{i t Z_{m p}}-\prod_{j=1}^{m} E e^{\frac{i t}{\sqrt{m}} W_{j}^{p}}\right| & \leqslant C t^{2} \sum_{k=1}^{q}\left|\sigma_{p}^{2}\left(x_{k}, y_{k}, x_{k}, y_{k}\right)-\sigma^{2}\left(x_{k}, y_{k}, x_{k}, y_{k}\right)\right| \\
& +2 t^{2} \sum_{k=1}^{q-1} \sum_{l=k+1}^{q}\left|\sigma_{p}^{2}\left(x_{k}, y_{k}, x_{l}, y_{l}\right)-\sigma^{2}\left(x_{k}, y_{k}, x_{l}, y_{l}\right)\right| .
\end{aligned}
$$

Proof. This is a convergence of Newman's inequality (1984) and asymptotic properties of the variances of $Z_{\tilde{n}}$ and of $W_{j}^{p}$ mentioned above.

Lemma 8. For $p$ fixed, we have

$$
\left|\prod_{j=1}^{m} E e^{\frac{i t}{\sqrt{m}} W_{j}^{p}}-e^{\frac{t^{2} \sigma_{p}^{2}}{2}}\right| \longrightarrow 0 .
$$


Proof. Just apply the Lindeberg condition to the variables $m^{-1 / 2} W_{j}^{p}, j=$ $1, \ldots, m$ and take account of the fact that each $W_{j}^{p}$ is a sum of $p$ bounded variables, so it is also bounded.

Now by summarizing the results, we have the asymptotic normality in the next theorem which can be obtained from Lemmas 6, 7 and 8. The proof is similar to the proof of Theorem 5.5 of Azevedo and Oliveira (2000) and, therefore is omitted.

Theorem 5. The random vector $\left(\alpha_{n}\left(x_{1}, y_{1}\right), \ldots, \alpha_{n}\left(x_{q}, y_{q}\right)\right)$ converges in distribution to a normal centered vector with covariance matrix

$$
\sum=\left[\begin{array}{cccc}
\sigma^{2}\left(x_{1}, y_{1}, x_{1}, y_{1}\right) & \sigma^{2}\left(x_{1}, y_{1}, x_{2}, y_{2}\right) & \ldots & \sigma^{2}\left(x_{1}, y_{1}, x_{k}, y_{k}\right) \\
\sigma^{2}\left(x_{2}, y_{2}, x_{1}, y_{1}\right) & \sigma^{2}\left(x_{2}, y_{2}, x_{2}, y_{2}\right) & \ldots & \sigma^{2}\left(x_{2}, y_{2}, x_{k}, y_{k}\right) \\
\ldots & \ldots & \ldots & \ldots \\
\sigma^{2}\left(x_{k}, y_{k}, x_{1}, y_{1}\right) & \sigma^{2}\left(x_{k}, y_{k}, x_{2}, y_{2}\right) & \ldots & \sigma^{2}\left(x_{k}, y_{k}, x_{k}, y_{k}\right)
\end{array}\right]
$$

\section{References}

Alam, K. and Saxena, K.M.L. (1981). Positive dependent in multivariate distributions, Commun. Statist-Theor. Math., 10, 1183-1196.

Azevedo, C. and Oliveira, P.E. (2000). Kernel-type estimation of bivariate distribution function for associated random variables. In New Trends in Probability and Statistics, 17-25.

Donsker, M.D. (1951). An invariant principle for certain probability limit theorems. Mem. Amer. Math. Soc., 6.

Henriques, C. and Oliveira, P.E. (2003). Estimation of a two dimensional distribution function under association. J. Statist. Planning Inf., 113, 137-150.

Jabbari, H., Azarnoosh, H.A. and Fakoor, V. (2009). Almost sure convergence of twodimensional distribution function under negative association. Journal of Applied Probability ES Statistics, 4, 157-166.

Joag-Dev, K. and Proschan, F. (1983). Negative association of random variables, with application. Ann. Statist., 11, 286-295.

Liang, H.Y. and Su, C. (1988). Convergence in the law of logarithm for NA sequences. Chinese Sci. Bull., 43, 1919-1925 (in Chinese).

Matula, P. (1992). A note on the almost sure convergence of sums of negatively dependent random variables. Statist. Probab. Lett., 15, 209-213. 
Newman, C.M. (1980). Normal fluctuations and the FKG inequalities. Comm. Math. Phys., 74, 119-128.

Newman, C. (1984). Asymptotic independence and limit theorems for positively and negatively dependent random variables, in: Inequalities in Statistics and Probability, Y. L. Tong, ed, IMS Lecture Notes- Monograph Series, 5, 127-140.

Roussas, G.G. (1994). Asymptotic normality of random fields of positively or negatively associated processes. J. Multivariate Anal., 50, 152-173.

Sadikova, S.M. (1966). Two-dimensional analogies of an inequality of Esseen with applications to the central limit theorem. Theory Probab. Appl., 11, 325-335.

Shao, Q.M. (2000). A comparison theorem on maximal inequality between negatively associated and independent random variables. J. Theore. Probab., 13, 343-356.

$\mathrm{Su}, \mathrm{C}$. and Chi, X. (1998). Some result on CLT for nonstationary NA sequences. Acta. Math. Appl. Sinica, 21, 9-21.

Su, C., Zhao, L.C. and Wang, Y.B. (1997). Moment inequalities and weak convergence for negatively associated sequences. Science in China (series A), 40, 172-182.

Yuan, M., Su, C. and Hu, T. (2003). A central limit theorem for random fields of negatively associated process. J. Theore. Probab., 16, 309-323.

H. Jabbari Nooghabi

Department of Statistics,

Ordered and Spatial Data Center of Excellence,

Ferdowsi University of Mashhad,

Mashhad, Iran.

email: Jabbarinh@um.ac.ir 